\title{
Numerical analysis of temperature effect on spall in natural uranium
}

\author{
O.A. Tyupanova ${ }^{a}$ and S.S. Nadezhin \\ Russian Federal Nuclear Center-VNIIEF, Sarov, Russia
}

\begin{abstract}
The work presents the modification of two-stage kinetic model of spall fracture (NAG model type) concerning the effect of temperature and strain rate on its parameters. It is shown that the proposed modification extends the model's range of convenience significantly.
\end{abstract}

\section{Introduction}

Among the fundamental properties of matter, which should be taking into account under shock wave loading and subsequent release waves, there are, first of all, resistance for plastic deformation (shear strength) and resistance for impulse tensile stresses (spall strength). Model of relaxational elasticplastic medium called "RING" [1] has been developed and is widely used at VNIIEF to describe first of these properties.

The most perspective approach to describe the spall fracture, used at VNIIEF, is presented by the two-stage micro-statistical kinetic model (NAG model type) [2,3]. The model possesses six parameters, which are usually determined by using experimental data. These parameters were considered as constant values, being the characteristics of each specific material, in the fundamental work [2] and till nowadays. If analyzing the physical sense of these parameters, it becomes obvious that they could depend on characteristics of stress-strain state and matter properties (such as grain size), and manufacturing procedure as well.

\section{Modification of the model}

\subsection{The simulation procedure}

The numerical analysis of the effect of stress-strain state characteristics onto the spall was performed by DRAKON numerical code [4]. Spherical component of the stress tensor for natural uranium was in the form of Mie-Gruneisen EOS with the Gruneisen's coefficient $\Gamma_{p}=\Gamma_{p}(\delta)$, depending on compression. To simulate the high-strain-rate deformation we used RING model [1]:

$$
\begin{gathered}
\begin{cases}\dot{Y}_{d}=3 G \dot{\varepsilon}_{i}-\frac{Y_{d}-Y_{S}}{\tau}, & Y_{d}>Y_{S} \\
\dot{Y}_{d}=2 G \dot{\varepsilon}_{i}, & Y_{d}<Y_{S}\end{cases} \\
Y_{s}=Y_{0} \cdot\left[1+a_{1} \cdot\left(1-\bar{T}^{a_{3}}\right) \cdot\left(1-\exp \left(-a_{2} \cdot \varepsilon_{i}^{p}\right)\right)\right] \cdot\left[1+a_{4} \cdot P_{x}\right] \cdot\left[1-a_{5} \cdot \bar{T}^{a_{6}}\right]
\end{gathered}
$$

\footnotetext{
a e-mail: root@gdd.vniief.ru; tyupanova_oa@ifv.vniief.ru
}

This is an Open Access article distributed under the terms of the Creative Commons Attribution-Noncommercial License 3.0, which permits unrestricted use, distribution, and reproduction in any noncommercial medium, provided the original work is properly cited. 
EPJ Web of Conferences

Table 1. Parameters of spall fracture model for natural uranium.

\begin{tabular}{|c|c|c|c|c|c|}
\hline$P_{n 0}, \mathrm{GPa}$ & $P_{g 0}, \mathrm{GPa}$ & $P_{1}, \mathrm{GPa}$ & $\dot{N}_{00}, \mathrm{~cm}^{-3} \cdot \mathrm{s}^{-1}$ & $\eta, \mathrm{Pa} \cdot \mathrm{s}$ & $R_{m=0}, \mathrm{~cm}$ \\
\hline 2.0 & 0.85 & 0.04 & $4.6 \cdot 10^{10}$ & 44.0 & $5.0 \cdot 10^{-5}$ \\
\hline
\end{tabular}

$$
\tau=\tau_{0} \cdot(1-\bar{T}) /\left(1+\dot{\varepsilon}_{i} / \dot{\varepsilon}_{i 0}\right)^{0.5}
$$

where $Y_{d}$ - is the effective yield strength, $G-$ is the shear modulus, $G=3 \cdot(1-2 v) \cdot \rho \cdot C_{B}^{2} /[2 \cdot(1+v)]$, $\dot{\varepsilon}_{i}-$ is the strain rate intensity, $\tau$ - is the elastic stresses' relaxation time, $Y_{s}-$ is the stationary yield strength; $a_{1} \ldots a_{6}, \tau_{0}$ and $\dot{\varepsilon}_{i 0}-$ are parameters, determined during the verification of the model, $\bar{T}=$ $T / T_{m}$ is the relative temperature, $T_{m}$ - is the melting temperature in a specific stress-strain state, calculated by Lindeman's law.

The two-stage kinetic model under study [2] considers the fracture as nucleation and growth of damage in the shape of micro-defects, distribution of which complies with statistical law. The main equations of the model in case of viscous fracture are presented below:

$$
\begin{gathered}
N_{m}(R)=N_{0} \exp \left(-R / R_{m}\right) \quad \text { - distribution of defects } \\
\frac{\mathrm{d} N_{0}}{\mathrm{~d} t}=\dot{N}_{00} \cdot \exp \left(\frac{P-P_{n 0}}{P_{1}}\right) \Theta\left(P-P_{n 0}\right) \quad \text { - nucleation of defects } \\
\frac{\mathrm{d} R_{m}}{\mathrm{~d} t}=\frac{P-P_{g 0}}{4 \eta} \cdot R_{m} \cdot \Theta\left(P-P_{g 0}\right) \quad \text { - growth of defects }
\end{gathered}
$$

In equations (4)-(6): $N_{0}$ is the total number $/ \mathrm{cm}^{3}$ of voids, $N_{m}$ is the cumulative number $/ \mathrm{cm}^{3}$ of voids with radii larger than $R_{m}, R$ is the void radius, $R_{m}$ is the parameter of distribution, $\dot{N}_{00}, P_{n 0}, P_{1}, P_{g 0}$, $\eta$ are constants; $P_{n 0}$ is the threshold for nucleation of voids; $P_{g 0}$ is the threshold for growth of voids, $\eta$ is the effective viscosity; $\Theta$ is the unit function. Parameters of this model for natural uranium (see Table 1) were verified under simulation of significant volume of experimental data on spall fracture with variation of loading intensity and symmetry. Till nowadays these parameters were considered as constants.

\subsection{Modification of the spall fracture model}

Any modification of a model of phenomenological character should be obviously based on laws revealed from special-purposed experiments. The most experimental studies of the effect of some factors upon the spall does usually result in stating relationships, which are mostly qualitative ones, between a spall strength $\sigma_{\text {spall }}$ (e.g., calculated from free surface curves) or a damage level (e.g., visually estimated) and changing of some factor or their group. In spite of the insufficient scope of experimental data, the clear physical meaning of six parameters of the model (4)-(6) allows us to take some effect into account directly. Such approach is illustrated in this work, taking two characteristics of stressstrain state as an example: preliminary heating and strain rate.

The first modification, proposed in this work, is based on the results of refs. [5,6], where it was obtained for some metals that the spall strength decreases insignificantly in a wide range of temperature and drops dramatically near the melting temperature. We used these data for stating the relationships between two thresholds - of damage nucleation and growth- and relative temperature

$\bar{T}$. The analytical form of the relations is written in the form below:

$$
P_{n 0}^{\prime}=P_{n 0}+\frac{P_{n 0}^{m}-P_{n 0}}{1+m_{1} \cdot \bar{T}^{m_{2}}}
$$




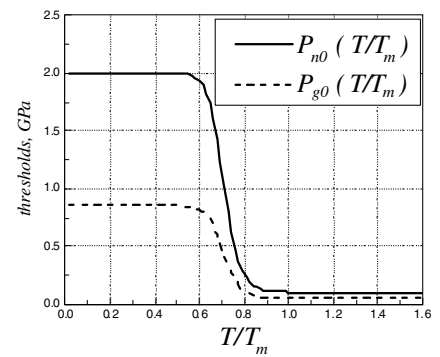

Fig. 1. Thresholds $P_{n 0}$ and $P_{g 0}$ vs relative temperature (eqs. (7)-(8)).
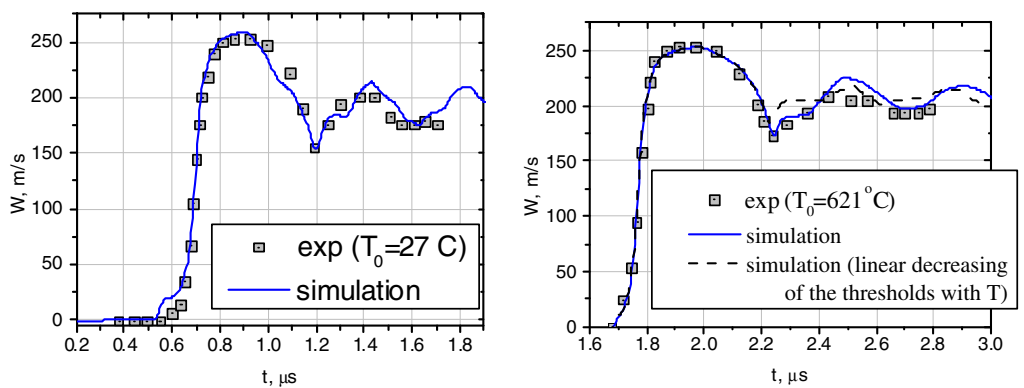

Fig. 2. Comparison of experimental [8] (VISAR) and numerical velocities of free surface $W(t)$.

$$
P_{g 0}^{\prime}=P_{g 0}+\frac{P_{g 0}^{m}-P_{g 0}}{1+m_{1} \cdot \bar{T}^{m_{2}}},
$$

where $m_{1}=0.001, m_{2}=-20-$ are constant values, $P_{n 0}^{m}=0.1 \mathrm{GPa}, P_{g 0}^{m}=0.05 \mathrm{GPa}-$ are the damage nucleation and growth thresholds in melting state. The relations (7)-(8) are also shown in Fig. 1.

Some experimental data available show that the viscosity of different character drops with increasing of temperature and strain rate. But these data are not enough to reveal an analytical dependence. So, based on the data from ref. [7], the effective viscosity is proposed to be written as:

$$
\eta=2 \cdot G \cdot \tau
$$

where $G$ is the shear modulus, $\tau$ is the elastic stresses' relaxation time, determined by eq. (3). The following condition is imposed on the viscosity: $\eta>\eta_{\min }$, where $\eta_{\min }$ is the least possible value of the viscosity. So, the viscosity is a decreasing function of two variables - temperature $T$ and strain rate $\dot{\varepsilon}$. Using equation (9) relates the high-strain-rate model with spall fracture model directly.

\section{Results and discussion}

The correctness of the proposed shape of the relationships between the damage nucleation and growth thresholds and temperature is confirmed by data, presented in Fig. 2, where experimental free surface curves $W(t)$ from Ref. [8] are compared with the results of numerical simulation. Experimental curves in [8] were obtained for pure uranium under room temperature and after pre-heating up to different temperatures. We compared our numerical curves with two experimental ones, which were obtained for $\alpha$-phase of pure uranium (heating below $\alpha-\beta$ phase transition).

One can see from Fig. 2, that it is precisely these dependencies $P_{n 0}(\bar{T}) \& P_{g 0}(\bar{T})$ (eqs. (7) and (8)), that provide a proper quantitative description of experimental data by numerical simulation. If using another type of these dependencies, e.g. linear decreasing of the thresholds with temperature, there is no agreement between experiment and simulation (see Fig. 2(b), dash curve). 

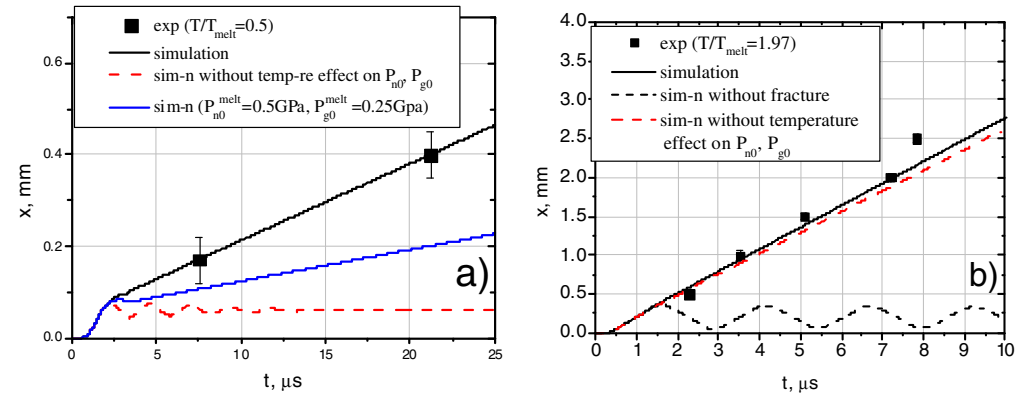

Fig. 3. Expansion of natural uranium under pulse heating. Experiment vs numerical simulation.

To verify the proposed modification of spall fracture model, the results, obtained at RFNC-VNIIEF, on natural uranium free surface movement measured by electric pins have been used. The samples of natural uranium were subjected to high-speed neutron heating up to different levels of introduced energy (method of "thermal shock"). A comparison of numerical curves $x$ - $t$ (solid) and experimental points in Fig. 3 illustrates the necessity to take into account temperature effect on spall fracture under simulating these experiments. Otherwise, numerical curves (red) do not repeat the position of experimental points.

Moreover, as it is seen from Fig. 3(a), these tests allows one to verify the extreme values of the damage nucleation and growth thresholds in melting state $-P_{n 0}^{m}$ and $P_{g 0}^{m}$ : increased values, as compared to that one's accepted in eqs. (7)-(8), results in a drastic shift of numerical curve (blue curve in Fig. 3(a)). The similar result has been obtained, concerning the effective viscosity $\eta$. If taking $\eta=$ const, as mentioned in Table 1, the numerical curve is much higher than experimental points.

\section{Conclusion}

A simple way how to modify the two-stage kinetic model has been demonstrated in this work. The appropriateness of taking into account the effect of temperature and strain rate onto parameters of the two-stage spall fracture model has been shown. In this work, three parameters among six ones - the thresholds for damage nucleation and growth, and the effective viscosity - are not constant, being the functions of stress-strain state characteristics. The proposed modification widens the range of convenience of the model significantly.

\section{References}

1. S.S. Nadezhin, O.A. Tyupanova, V.A. Raevsky, et al., New Models and Hydrocodes for Shock Wave Processes in Condensed Matter (Lisbon, Portugal, 2008)

2. L. Seaman, D. Curran, A. Shockey, J.Appl.Phys. 47, 4814 (1976)

3. O.N. Ignatova, O.A. Tyupanova, et al., VII Khariton's Scientific Readings (Sarov, Russia, 2005).

4. P.N. Nizovtsev, et al., IV Zababakhin's Scientific Readings (Snezhinsk, Russia, 1995)

5. G.I. Kanel, S.V. Razorenov, A.A. Bogatch, et al., J.Appl.Phys. 79, 8310 (1996)

6. R.M. Schmidt, F.W. Davies, B.M. Lempriere, et al., J.Phys.Chem.Solids. 39, 375 (1978)

7. D. Steinberg, D.Breithaupt, et al., Physica. 139\&140B, 762 (1986)

8. E. Zaretsky, B. Herrmann, D. Shvarts, SCCM (AIP Conference Proceedings, 2005) 292 (2006). 